\title{
Intelligent Information Technology in Education
}

\author{
Anna Yankovskaya $^{(1,2,3,4,5)}$, Yury Dementyev ${ }^{(3)}$, Danil Lyapunov ${ }^{(3,6)}$, Artem Yamshanov $^{(4)}$ \\ ${ }^{(1)}$ Tomsk State University of Architecture and Building \\ ${ }^{(2)}$ National Research Tomsk State University \\ ${ }^{(3)}$ National Research Tomsk Polytechnic University \\ ${ }^{(4)}$ Tomsk State University of Control Systems and Radioelectronics \\ ${ }^{(5)}$ Siberian State Medical University \\ ${ }^{(6)}$ Scientific Research Institute of Automation and Electromechanics of \\ Tomsk State University of Control Systems and Radioelectronics \\ Tomsk, Russia \\ ayyankov@gmail.com,dementev@tpu.ru, lyapdy@gmail.com, yav@keva.tusur.ru
}

\begin{abstract}
Intelligent information technology (IIT) for the purpose of education within the blended learning paradigm is discussed. The technology is based on the construction of mixed diagnostic tests (MDTs) used for decision-making. Each MDT represents a compromise between unconditional and conditional components. The cognitive graphic tools are used as IIT subsystem for decision justification. The IIT is proposed for control, students' knowledge monitoring, professional and personal skills and abilities development, and designing the learning trajectory for every student. A technique for IIT construction based on the MDTs, threshold and fuzzy logics, and cognitive graphic tools is suggested.
\end{abstract}

Keywords-Intelligent information technology, pattern recognition, mixed diagnostic tests, learning trajectory, blended learning, decision-making, cognitive graphic tools

\section{INTRODUCTION}

In recent years, the problem of intelligent information technologies is quite relevant for a number of subjects, especially in education [1-4]. Application of the mixed diagnostics tests (MDTs) $[3,4]$ is a promising approach to guide students towards the goals of a particular course. MDTs are a compromise between unconditional and conditional components. Construction of the MDTs represents the advanced way for students' learning and personal development.

The blended learning paradigm [5] is a relatively new educational technology. Despite its novelty for learning and training any educator should know that the blended education technologies have always been the key point at a higher school.

Blended learning represents an integrated training environment, which combines the advantages of e-learning and traditional classroom activities [2]. One of the main problems of a higher school is to develop mechanisms for effective education and to train a large number of graduates who will be able to solve different problems [3].

Variety of information technologies including methods of artificial intelligence are not widely used in the interaction process of a teacher and a student for the diagnostics of

Russian Foundation for Basic Research, project №16-07-00859, project №14-07-00673 (partially). acquired knowledge. Development of such systems requires considerable efforts, time and costs $[4,6]$.

Students of different abilities have diverse preferences in process of learning and reaching their goals. L. Ausburn [7] used a special questionnaire aimed to estimate the students' abilities such as initial level of their knowledge, skills, and experience. Orientation on a student's particular interests and capabilities make the learning process more effective and less time-consuming.

Any specialist should rely on experience and skills obtained at a higher school to find the proper solution of educational and industrial problems. Moreover, he should understand very clearly how the obtained knowledge, professional and personal skills, and abilities would be used to reach the goals.

At their research [5] Bliuc et al. identifies four different ways of the blended learning definition. Blended learning can be seen as: 1) a mix of modes of web-based technology, 2) a mix of various pedagogical approaches, 3) a combination of any form instructional technology with face-to-face instructor led training, 4) a combination of instructional technology with actual job tasks (in order to create an effective mix of learning and working).

The essential component of any intelligent technology is decision-making tool. The tool can be created on the basis of threshold and fuzzy logics, and cognitive graphic tools [4].

Fuzzy set theory was suggested by Lotfi Zadeh in 1965 and developed in [9]. Fuzzy logic has been applied in many problematic areas from control theory to artificial intelligence. Fuzzy logic is a form of many-valued logic. In contrast to traditional logic theory where two-valued variables (true or false) are used, fuzzy logic variables may have the truth value within the range between 0 and 1 .

One of the new directions in pattern recognition with using fuzzy logic is represented in the papers of L. Zadeh [9], V. Novak, I. Perfilieva [10], I. Batyrshin [11], V. Tarasov, A. Averkin, N. Yaroushkina, I. Astachova [9-11]. The constructed models using fuzzy expert evaluations and fuzzy systems are the bases of this direction. 
Promising researches on combining threshold and fuzzy logics to implement the pattern recognition and knowledge testing are represented in [12].

A valuable step in the assessment and self-assessment process is the use of cognitive graphic tools [13]. They are able to give all appropriate information about the learning process both in statics and dynamics. Moreover, these tools motivate the students to create their own learning trajectories, guiding them to learn again and clarify the misunderstood material if it is necessary. As a result, the students are able to achieve a higher level of professional development in learning the discipline.

In this paper the intelligent information technology (IIT) based on the blended learning approach, the MDT construction, threshold and fuzzy logics, and cognitive graphic tools is presented. The IIT provides the formation of individual learning trajectory design, which helps the students to advance towards the course's goals.

For effective IIT implementation the following problems should be solved: a) offering students the instrument for designing their own learning trajectories, b) providing interaction between a teacher and a student, and c) providing access to appropriate information blocks. The educational process is expected to be much more effective and less timeconsuming as a result of the approach suggested.

\section{PROBLEM BACKGROUND}

The modern society involves dynamic, frequently unpredictable changes, which motivate students and graduates to be able to solve different educational and professional problems [14]. It should be noted that the blended learning process requires an extension of skills range, experience and knowledge (competences) of both teachers and students.

The MDTs represent a versatile means to enhance and develop students' skills and abilities. Essentials of the MDTs used in intelligent learning and training systems are fully outlined in the paper [15].

Diagnostic test is a set of grouped characteristic features. Unconditional component of the diagnostic test is characterized by the simultaneous presentation of all its constituent features to the object (respondent, student) during the decision-making. Conditional component of the diagnostic test is characterized by the sequential presentation of features, depending on the values of the previous features.

The problem may be formulated as follows. An educational course is usually divided into some sections (modules). Each of those consists of didactic units. For the correct IIT design, it is required to construct the MDTs for each module of the course and to make decision using the results of the MDTs estimated via threshold and fuzzy logics, and cognitive graphic tools.

\section{LEARNING TRAJECTORY DESIGN OF COURSE USING MDTS}

Learning trajectory design of a course using the MDTs will be illustrated on the example of the MDT construction for the university course "Information Technology" for specialization "Industrial and Civil Construction" [12]. The main sections (modules) of the course are as follows: 1) the concept of information; 2) a general description of the data collection, transmission, processing and accumulation of information; 3) hardware and software implementation of information processes; 4) decision models of the functional and computational tasks; 5) algorithms and programming; 6) high level programming language; 7) database; 8) software and programming technology; 9) computer graphics; 10) computer practice. Each grouped characteristic feature contains a different number (from 4 to 8 ) of didactic units (characteristic features).

An unconditional component of the MDT includes grouped characteristic features of sections 1-3. Note that grouped characteristic features (e.g. for section 3) should be divided into: "3.1. The technical tools to implement information processes" and "3.2. Software implementation of information processes". A conditional component of the MDT includes grouped characteristic features of 3.2, 4-9.

An example of the MDT search tree is given in Figure 1. The numbers of the course sections are written in nodes (represented by rectangles). Objects assigned with the unconditional component of the MDT (sections 1, 2 and 3.1) are listed in the root node of the tree. Transition to knowledge control on course sections, which relate to the conditional component of the MDT is provided if the student has successfully completed the task on the unconditional component of the MDT and scored at least 50 percent of the correct answers. Branches of tree are marked by letters $a, b$, and $c$ on arcs coming out of the root node (Figure 1).

A possibility to skip of a section is excluded during the construction of the MDT and the students are invited to pass the section, which is connected with subsequent sections. Full sequence of branches tree search for the section 3.2 is outlined in Figure 1. Thereafter the student may proceed to carry out assignments from section 4 or 5 . Similarly, while performing the tasks for sections 4 and 5 he can proceed to implement the tasks of the sections 3.2 and 5 or 3.2 and 4 respectively.

One of the possible variants of the conditional component of the test is the following admissible sequence: $3.2,4,5,6,8$, 7, 9. Applied part of course from section 10 may be indirectly presented in each of the sections 1-9 as practical tasks. The tree leaves are shown as ovals. The test results were compared to the tree leaves. The tree edges with dashed arrows indicate the

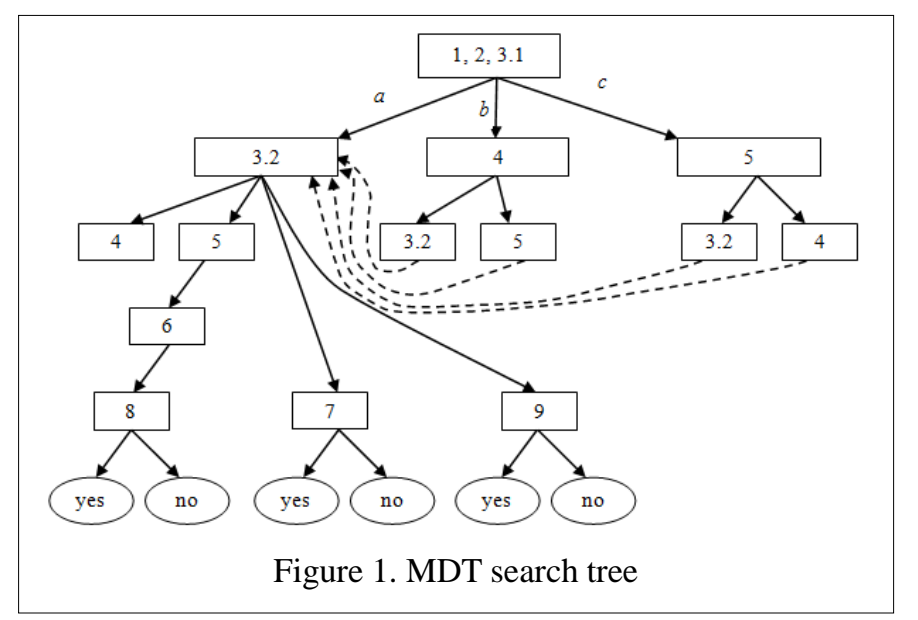


possible transitions of passing a conditional component of the MDT.

We have a sequence of different courses and sections (topics) for each course. The number of courses during a term is defined by an expert (or by a group of teachers). The complexity of courses based on credits of each courses. A student can choose a form of education and training from the different approaches and resources. Decision-making about a student's level of knowledge, professional and personal skills and abilities is carried out using threshold and fuzzy logics [16].

\section{COGNITIVE GRAPHIC TOOLS}

Firstly, transformation of features space in patterns space based on the logical-combinatorial methods and properties of n-simplex are suggested in the publication [17]. System of visualization TRIANG for decision-making and its justifications with cognitive graphic tools [18] is constructed on the basis of the theorem proved by A. Yankovskaya [17]. This theorem was used in more than 30 applied intelligent systems and in three tools of revealing different kinds of regularities and decision-making of diagnostic, organizational and control, classification types, and their justifications.

The main function of $n$-simplex is a representation of a disposition of object under study among other objects of a learning sample [19]. Additionally, n-simplex has other useful functions for a decision-making person. One of these functions is a representation of some numerical values, for example, an admissible error of recognition preassigned by the user. An example of 2-simplex cognitive graphic tool is shown in Figure 2.

The sides of 2-simplex (triangle edges) are associated with patterns (classes), circles with big radius are the objects under study and circles with small radius are learning sample objects. In our case, the patterns are satisfactory, good and excellent evaluations of student's learning result. The distance from the object to a side is directly proportional to the proximity of the object to the pattern corresponding to the side. The distance for object under study is displayed as color perpendicular lines to 2 -simplex sides (red, yellow, green). The color of the object

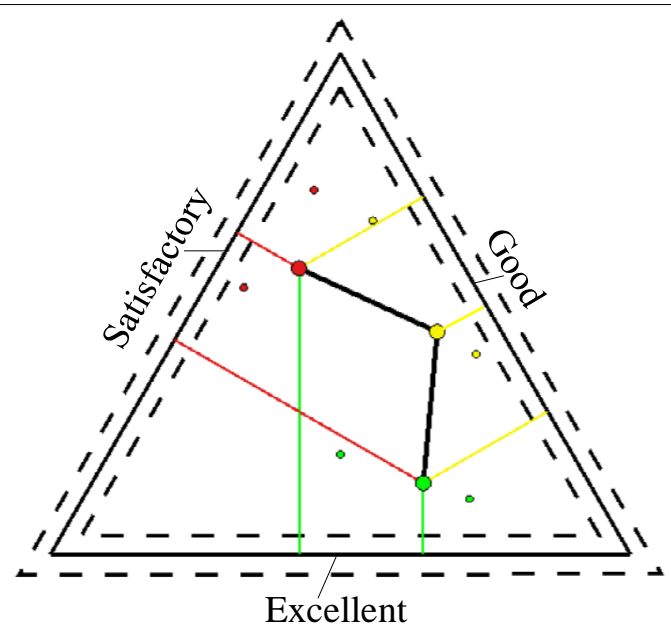

Figure 2. 2-simplex cognitive graphic tool under study or objects from learning sample is mapped to a pattern, which is revealed for a specific object. An object color is mapped with an associated pattern (the nearest pattern or pattern determined by an expert).

The cognitive graphic tool "2-simplex prism" (Figure 3) is based on the 2-simplex and represents the equilateral triangular prism which contains in the cross-sections 2-simplexes which correspond to the fixed time moments.

The distance from the prism base to $i$-th 2 -simplex $h_{i}{ }^{\prime}$ corresponds to the fixed time moment of the object's features and it is calculated based on the following formula:

$$
h_{i}{ }^{\prime}=H^{\prime} \cdot \frac{T_{i}-T_{\min }}{T_{\text {max }}-T_{\min }},
$$

where $H^{\prime}$ is a length of 2-simplex prism preassigned by a user and corresponded to the duration of learning, $T_{i}$ is a timestamp of features fixation of object under study for $i$-th examination, $T_{\min }$ is a timestamp of features fixation of the object for the 1st examination, $T_{\max }$ is a timestamp of features fixation of the object for the last examination.

The dashed line shown in Figure 3 corresponds to the individual learning trajectory for a student, based on his/her learning results.

2-simplex prism allows representing visually the dynamic processes within the problematic area. That is necessary for a big amount of problems and such cross-disciplinary areas as medicine, economics, genetics, building, radioelectronics, sociology, education, psychology, geology, design, ecology, geo-ecology, eco-bio-medicine, etc.

When it is necessary to use four patterns the 3-simplex cognitive graphic tool application is appropriate.

Figure 4 shows learning dynamics for the three respondents. We assume that the first result (lower red point) corresponds to the first testing at the beginning of the course,

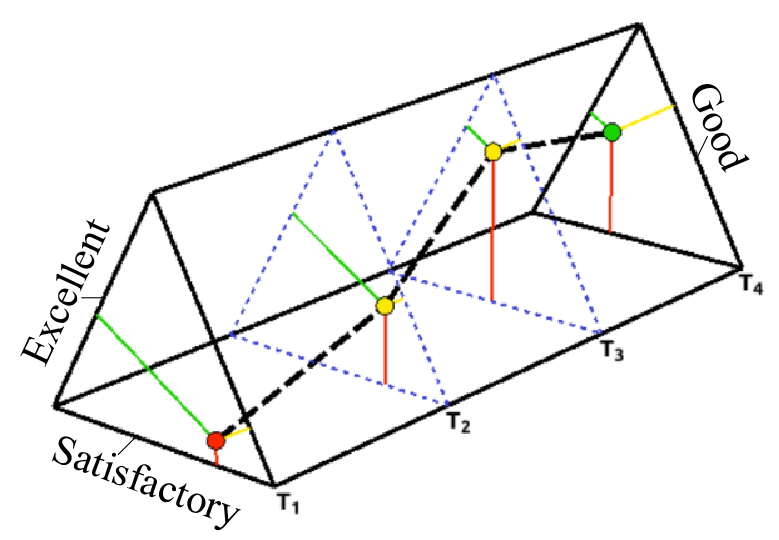

Figure 3. 2-simplex prism cognitive graphic tool 
where all of the respondents showed the same unsatisfactory result. The second result corresponds to testing in the middle of the course. It clearly shows the learning speed differentiation of the respondents: the respondents $a$ and $b$ showed approximately the same learning speed at the beginning of the course, while the respondent $c$ demonstrates a faster learning speed (the pursuit to excellent evaluation, ability to learn). Due to the information obtained, it is possible to perform the next actions.

1. Forecasting of further knowledge level increase of respondents.

2. Subdividing the respondents into different groups corresponding to various degrees of learning ability, and suggesting them the appropriate tasks. Such an approach would increase the level of knowledge among the respondents dividing them into groups. Splitting the respondents into groups is both economic and less time-consuming than the individual training of each respondent.

The third result corresponds to respondents' testing after finishing the course. Figure 4 shows that the respondents $b$ and $c$ have acquired excellent marks, but the respondent $c$ showed more reliable knowledge and a higher development potential than the respondent $b$. This fact can be correctly forecasted with high reliability from the dynamics, which we have after the second test.

The respondent $a$ passed the test with a good mark, and he has prepared for the test once more and made another attempt, showing a little progress. This fact could be forecasted from the dynamics of the passage of the previous tests with high reliability.

The essential advantages of the 3-simplex use for IIT are as follows:

1. High visibility of the learning dynamics outcomes and the ability to compare the learning dynamics for different

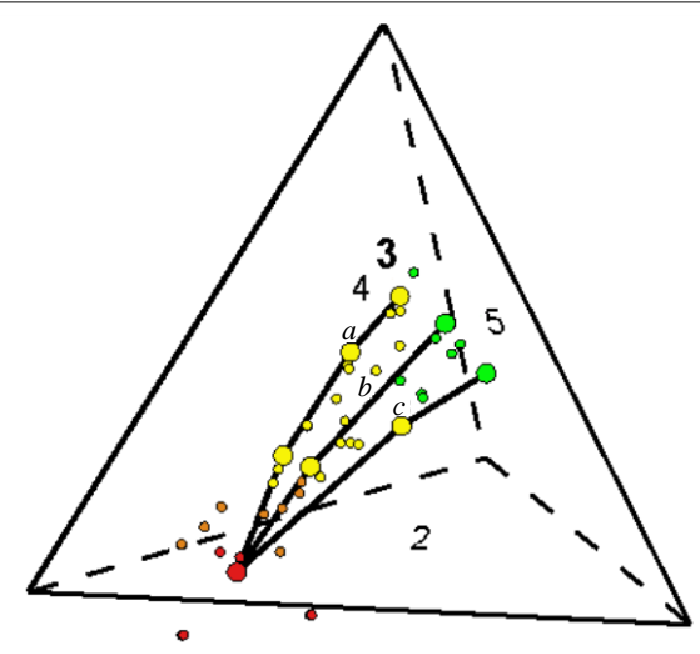

Figure 4. Learning dynamics for the three respondents visualized by the 3 -simplex cognitive graphic tool respondents.

2. Taking weight coefficients into consideration for different types of questions allows a more reliable evaluation of the test results.

3. "Abnormal" results detection, i.e., the results obtained via cheating or randomly guessing.

4. Detection and mapping of the degree of the respondent's ability to learn.

\section{CONCLUSION}

The IIT based on the MDTs, threshold and fuzzy logics, and cognitive graphic tools is proposed. The IIT allows improving the quality of a learning process and the accuracy of its assessment. The original practice of the MDTs application and further assessment of the learning results, which allows reducing significantly the costs and time for learning and training the students while increasing the quality of education, is shown.

The background, the formulation of the problem and the method of the MDTs construction are given. The proposed IIT is also aimed at students' motivation on studying the sections (modules) during a term, thus, forming the individual learning trajectory for each student.

Threshold and fuzzy logics application increases accuracy and estimation quality of respondents (students). The blended education approach is applied for learning different courses, e.g., mathematics, information technology, geoinformation systems, mathematical modeling, electrical engineering, etc.

Cognitive graphic tools application for test results evaluation allows making and justifying decisions about learning results at fixed time moments.

The approach developed for the IIT increases effectiveness of blended learning by choosing the shortest ways to get the correct result and by excluding the possibility of achieving it at a random path.

2-simplex prism cognitive graphic tool allows to study objects dynamically within the time range interesting for a user. 3 -simplex is used for study the learning results related to 4 patterns.

It is planned to apply the IIT for the other courses such as discrete mathematics, electric drive theory, power electronics with different didactic units.

The IIT can be designed in such a way that it would serve as a guide to explain the procedure of solving complex practical problems for students and specialists in some problematic area.

\section{Acknowledgment}

The work is supported by Russian Foundation for Basic Research, project №16-07-00859, project №14-07-00673 (partially). 


\section{References}

1 P. Brusilovsky, J. Knapp, J. Gamper, Supporting Teachers as Content Authors in Intelligent Educational Systems, International Journal of Knowledge and Learning 2(3/4), 2006, 191-215.

2 V. Uskov, A. Uskov, Computers and Advanced Technology in Education - Perspectives for 2010-2015, Proc. of the 13th IASTED International Conference on Computers and Advanced Technology in Education, Maui, Hawaii, USA, 2010.

3 Yankovskaya, A. Mixed diagnostic tests as innovation approach in VLSI engineering education // 10th European Workshop on Microelectronics Education (EWME), 2014, pp. 219-223. IEEE.

4 A. Yankovskaya, Yu. Dementyev, D. Lyapunov, A. Yamshanov, Design of Individual Learning Trajectory Based on Mixed Diagnostic Tests and Cognitive Graphic Tools // Proceedings of the IASTED International Conference "Modeling, Identification and Control" MIC (MIC-2016) February 15-16, 2016 Innsbruck, Austria. - 2016, 59-65.

5 A.-M. Bliuc, P. Goodyear, R.A. Ellis, Research Focus and Methodological Choices in Studies into Students' Experiences of Blended Learning in Higher Education. Internet and Higher Education, 10, (2007), 231-244.

6 A. Yankovskaya, M. Semenov, Application Mixed Diagnostic Tests in Blended Education and Training // Proceedings of the IASTED International Conference Web-based Education (WBE 2013) February 13 - 15, 2013 Innsbruck, Austria. - 2013. pp. 935-939.

7 L. Ausburn, Course Design Elements Most Valued by Adult Learners in Blended Online Education Environments: An American perspective. Educational Media International, 41(4), (2004), 327-337.

8 A. Yankovskaya, M. Semenov, Foundation of the Construction of Mixed Diagnostic Tests in Systems for Quality Control of Education. Proc. 13th IASTED International Conference Computers and Advanced Technology in Education (CATE 2010) August 23-25, 2010, Maui, Hawaii, USA, 142-145.

9 L. Zadeh, Fuzzy logic and approximate reasoning. Synthese, 30, 1975, 407-428.
10 V. Novak, I. Perfilieva, J. Mockor, Mathematical principles of fuzzy logic, (Kluwer Academic Publishers, 1999).

11 I. Batyrshin, et al., Theory and practice of fuzzy hybrid systems, (Moscow, Fizmatlit, 2006).

12 A. Yankovskaya, M. Semenov, Computer Based Learning by Means of Mixed Diagnostic Tests, Threshold Function and Fuzzy Logic, Proc. of the IASTED International Conf. Human-Computer Interaction, Baltimore, USA, 2012, 218-225.

13 A. Yankovskaya, Decision-making and decision-justification using cognitive graphics methods based on the experts of different qualification, Russian Academy of Science Bulletin, Theory and Control Systems, 5, 1997, 125-128.

14 F. Singera, D. Stoicescu, Using Blended Learning as a Tool to Strengthen Teaching Competences. Procedia Computer Science. 3, (2011), 1527-1531.

15 A. Yankovskaya, Mixed Diagnostic Tests are a New Paradigm of Construction of Intelligent Learning and Training Systems. Proc. New quality of education in the new conditions. Tomsk, Russia, (2011), V.1., 195-203 (in Russian).

16 A. Yankovskaya, M. Semenov, Intelligent system for knowledge estimation on the base of mixed diagnostic tests and elements of fuzzy logic, Proc. of the IASTED International Conf. Technology for Education, Dallas, USA, 2011, 108-113.

17 A. Yankovskaya Transformation of features space in patterns space on the base of the logical-combinatorial methods and properties of some geometric figures. Proceedings of the International Conference Pattern Recognition and Image Analysis: New Information, Abstracts of the I All-Union Conference, Part II, pp. 178-181, Minsk, 1991.(in Russian).

18 S. Kondratenko, A. Yankovskaya System of visualization TRIANG for decision-making justification with cognitive graphics usage. Proceedings of the Third Conference on Artificial Intelligence. Vol. I. - Tver, 1992. p. 152-155 (in Russian).

19 A. Yankovskaya, A. Yamshanov, Family of 2-simplex cognitive tools and their applications for decision-making and its justification, Computer Science \& Information Technology (CS \& IT), 2016, 63-76. 